\title{
Toxicological effect of ethanol on the development of chicken embryo in ova
}

\author{
Gulnaz Afzal ${ }^{1 *}$, Sadaqat Ali ${ }^{1}$, Shumaila Kiran ${ }^{2}$, Ghulam Mustafa ${ }^{3}$, Hafiz \\ Ishfaq Ahmad ${ }^{4}$, Sajid Hameed ${ }^{5}$, Maimoona Nazir ${ }^{1}$, Rida Amjad ${ }^{1}$, Hafiz \\ Muhammad Arshad ${ }^{6}$, Tahira Ruby ${ }^{1}$ and Jafar Majeed ${ }^{1}$
}

1. Department of Zoology, The Islamia University of Bahawalpur, Bahawalpur-Pakistan

2. Department of Applied Chemistry, Government College University, Faisalabad-Pakistan

3. Department of Biochemistry, Government College University, Faisalabad-Pakistan

4. Guangdong Key Laboratory of Animal Conservation and Resource Utilization, Guangdong Public Laboratory of

Wild Animal Conservation and Utilization, Guangdong Institute of Applied Biological Resources, Guangzhou,

Guangdong-China

5. University College of Veterinary and Animal Sciences, The Islamia University of Bahawalpur, Bahawalpur-

Pakistan

6. Department of Chemistry, Government College University, Lahore-Pakistan

*Corresponding author's email: gulnaz.afzal@iub.edu.pk

\section{Citation}

Gulnaz Afzal, Sadaqat Ali, Shumaila Kiran, Ghulam Mustafa, Hafiz Ishfaq Ahmad, Sajid Hameed, Maimoona Nazir, Rida Amjad, Hafiz Muhammad Arshad, Tahira Ruby and Jafar Majeed. Toxicological effect of ethanol on the development of chicken embryo in ova. Pure and Applied Biology. Vol. 8, Issue 1, pp882-892.

http://dx.doi.org/10.19045/bspab.2019.80030

Received: 03/12/2018 Revised: 27/02/2019

Accepted: 06/03/2019

Online First: 15/03/2019

\section{Abstract}

This study aimed at finding out the toxicological effects of ethanol on chicken embryo during early stages of development. The nature and extent of toxicological effects were investigated to identify the concentration which causes the death of the embryo. The total $(n=32)$ eggs were divided into four groups viz. control group, group A, group B and group C. Eight eggs were placed in each group for incubation. Different concentrations of ethanol were given to those groups viz. 5\%, 10\%, and 15\% respectively. Gross anatomical defects particularly embryo's body weight, body length, wings length and hind limbs length throughout seven days incubation were measured after the exposure of mentioned ethanol concentrations. The results of mean values of seven-day-old chick embryos exposed to 5\% ethanol showed non-significant effect $(P>0.05)$ on body weight, wings length and the hind limbs length of all groups concerning control group chick embryo. But ethanol with a concentration of $10 \%$ and $15 \%$ showed a significant effect $(P<0.05)$ on the body length of chick embryo in comparison with $5 \%$ treated chicks. The results of hatchability showed that group A showed $90 \%$ normal hatchability at the $21^{\text {st }}$ day while group B showed no remarkable delay in comparison of group $\mathrm{C}$ which showed delayed hatching at $22^{\text {nd }}$ day due to exposure of heavy dose. Moreover, among the most frequent abnormalities, hind limb lengths at $15 \%$ and body weight at $5 \%$ were found reduced in size. Ethanol exposure increases the risks of developmental defects with increasing embryonic age, delayed and assisted hatching.

Keywords: Chicken; Embryo; Ethanol; Ova; Toxic

Introduction

Alcohol, despite being toxic to humans, is used in industry and home for the manufacturing of compounds like gasoline additives, paints, and perfumes. In general usage, alcohol refers almost always to ethanol, also known as grain alcohol. Ethanol is a colorless, inflammable compound, which 
is the component of alcohol containing beverages like wine, spirits, and champagne. Ethanol is also used as a solvent in medicines, perfumes, and vegetable essences, such as vanilla. It is also used as a topical antiseptic. Alcohol intake during pregnancy can lead to fetal abnormalities. Prenatal alcohol exposure exerts teratogenic effects on the developing fetus, mainly manifested by growth retardation, abnormal facial features, and Central Nervous System (CNS) damage, collectively known as the Fetal Alcohol Syndrome (FAS). Alcohol also causes cardiovascular anomalies [1]. Consumption of alcohol during pregnancy consequences of fetal alcohol syndrome (FAS), which include unprompted abortion, growth impedance, facial anomalies, and mental retardation. Alcohol produces its effects by narrowing of placental and umbilical cord blood vessels, decreasing the oxygen supply to the fetus. Placental measurements noticed from normal births demonstrated that umbilical blood vessels squeezed in the presence of alcohol even at concentrations as low as $0.05 \%$, the amount checked in 1 to 1.5 drinks. Oxygen deficiency may play a role in the development of FAS-associated with mental retardation and behavioral deficiencies [2]. Ethanol exposure accelerates the cells died within the area of cranial neural crest during embryogenesis, has been revealed to result in loss of the SHH gene (Sonic hedgehog) as well as transcripts involved in SHH signaling pathways. The SHH gene is responsible for the production of the Sonic Hedgehog protein which is an important morpho gene that shows a role in cell growth, cell specialization and the prefiguring of the body [3]. Thus, the CNS seems to be very susceptible to developmental exposure to alcohol across all stages of development with gross physical defects consequential from exposure at peri-organogenetic periods and insufficient in distinct neuronal populations when exposure takes place at the postnatal period or the end of gestation. Recently, with the introduction of new tools, variations in gene expression patterns associated with the above-stated neurulation or gastrulation phase, ethanol sensitive openings have initiated to develop new insight on the molecular mechanisms that involved in ethanol teratogenicity [4]. An essential issue in the research studies of developmental effects of alcohol is that the comparative roles of alcohol itself and that of its primary metabolite, acetaldehyde. The second is known to have teratogenic effects [5].

Furthermore, the maternal metabolic illness role of alcohol with or without placental dysfunction is undefined in the pathogenesis of FAS [6]. According to Gilbert, 2003, there is marked the difference in the capability of mothers and fetuses to metabolize alcohol, and it is supposed that $30-40 \%$ of the children will have FAS, born to mothers who drink alcohol during pregnancy [7]. Some of these complications can be overwhelmed by using the chick embryo, which does not metabolize alcohol to acetaldehyde until day 9 of gestation [8] and therefore permits the study of ethanol effects in the absence of its primary metabolite. The chick embryo is an attractive model to study developmental procedures. It has been used from so long as a replacement of human embryos due to their close biological similarity. In the early periods, chick embryo morphology is very analogous to human [9]. The study was aimed to investigate the effects of in ovo ethanol exposure with different concentrations on early developmental stages. The nature and magnitude of these effects were recorded to detect the concentration lethality which causes gross structural abnormalities along with their hatchability characteristics.

\section{Materials and methods}

Chick embryos were exposed to different ethanol levels and compared with same age controls. The study was carried out in the 
Laboratory of Zoology, The Department of Life Sciences, The Islamia University Bahawalpur, Bahawalpur Pakistan by the approval of the ethical committee.

\section{Handling of eggs}

A total of 32 fertilized, non-incubated, White Leghorn eggs (each weighing about 60g) were collected from Govt. poultry farm Bahawalpur, Punjab, Pakistan. All eggs were divided into four groups Viz. Control, A, B and $C$ having eight $(n=8)$ individuals in each. Four individuals from every group were sacrificed at day $7^{\text {th }}$ of development and compared with the same aged individuals of the control group. The remaining was kept constantly incubated till the day of hatching. The eggs were candled to pinpoint air cavity, and the unfertilized eggs were excluded from the experiments. Eggs were cleaned by $70 \%$ ethanol for aseptic measurements. To check the alcoholic teratogenicity in the chick, fertilized eggs were marked, numbered and divided into four groups. Including control, there were placed eight $(n=8)$ eggs in each group. A hole of diameter $2 \mathrm{~mm}$ in the center of air sac was made by egg driller in all eggs of all groups. The concentration of 5\%,10\% and $15 \%$ of ethanol was injected into the eggs of group A, group B, and group C respectively. The hole was sealed by using melted paraffin. All the eggs were left at room temperature in an upright position for 2 hours. After that, all were shifted to the incubator for incubation at $37^{\circ} \mathrm{C}$ with $55 \%$ relative humidity. Eggs which were cracked or stored in the refrigerator were excluded from the study. The total incubation period of chicken was 21 or 22 days.

\section{Examination of embryos}

Four embryos from every group at $7^{\text {th }}$ day were dissected and observed the effects of different concentrations of alcohol on total body weight, body length, wings length, and hind limbs length by cutting chorioallantoic membrane and amnion. Hatchability characteristics of the remaining four chicks from each respective group were also observed. Most of the chicks hatched at day 22 whereas the remaining chicks that could not hatch by natural way till day 22 were taken out manually by flouting the shells. The newly hatched chicks were inspected for gross anomalies, abnormal posture, gait and other abnormal behavior as compared to the control.

\section{Statistical analysis}

All ethanol-exposed the group's means and standard errors values regarding embryo's body weight, body length, wings length, and hind limbs length were calculated. The Tuckey's Multiple Comparative Test was used for post-hoc analysis. Percentage of newly hatched chicks with gross abnormalities and reduction of all alcoholexposed groups concerning particular developmental defects was examined.

\section{Results}

A total of 32 eggs were placed under experimental care those were divided into four (4) groups viz group A, group B, group $\mathrm{C}$, and control group. There were different concentrations of ethanol $00,5 \%, 10 \%$, and $15 \%$ were injected in that groups of eggs correspondingly to note its effect on body weight, body length, wings length and size of hind limbs length of chicks embryo. Measurements regarding body weight, body length, wings length, and hind limbs length were calculated at seven (7th) day of incubation while the other characteristics were noted on hatching at 21 st or 22 nd day.

\section{Effect of ethanol on body weight}

The results of weight according to the effect of different concentrations of ethanol are given in (Table $1 \&$ Figure 1). Its findings revealed that all the concentrations of ethanol showed no significant effect on the weight of chicks concerning the means values of control group individuals. 
Table 1. The Mean \pm SEM of body weight of $7^{\text {th }}$-day embryos at different levels of alcohol concentration

\begin{tabular}{|c|c|c|}
\hline \multicolumn{2}{|c|}{ Body Weight (grams) vs ethanol concentrations } \\
\hline Groups & Ethanol Concentration & SEM \\
\hline Control & 00 & $1.53 \pm 0.022$ \\
\hline A & $5 \%$ & $1.58 \pm 0.22$ \\
\hline B & $10 \%$ & $1.15 \pm 0.06$ \\
\hline C & $15 \%$ & $1.2 \pm 0.04$ \\
\hline
\end{tabular}

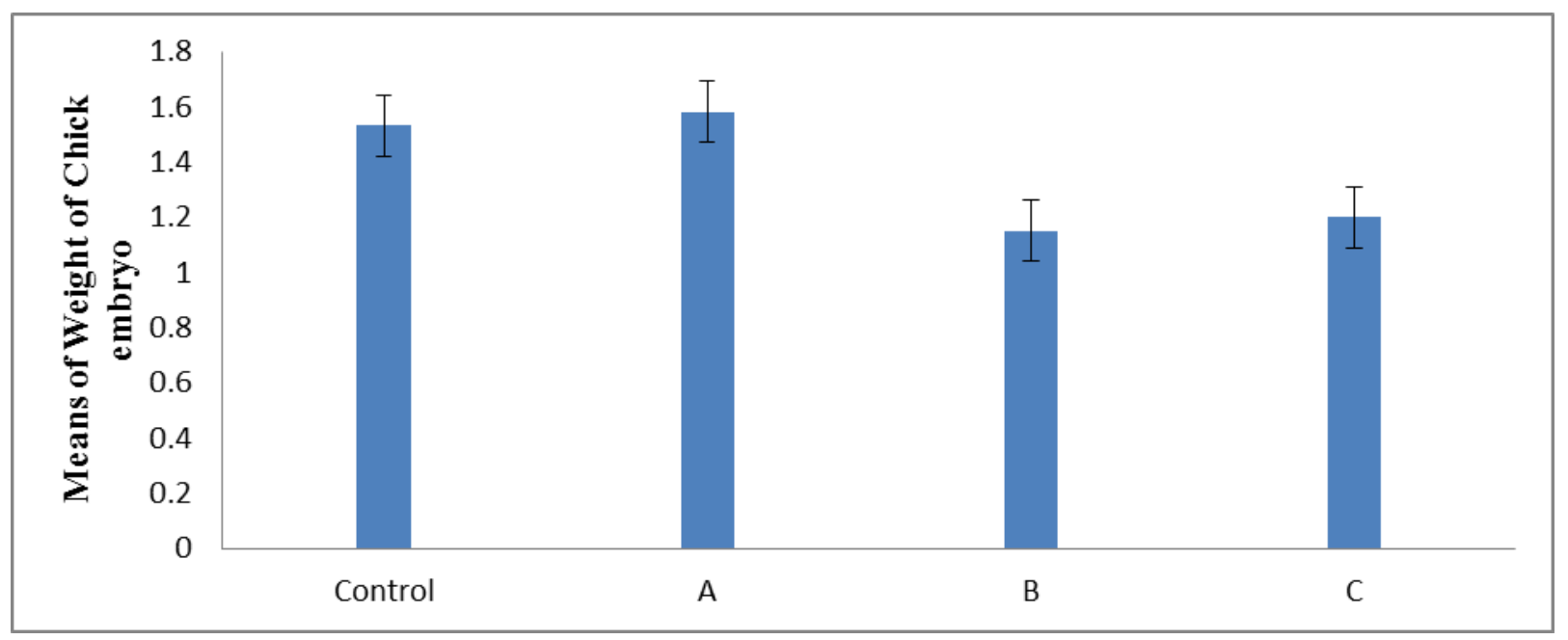

Figure 1. Comparison of Mean \pm SEM of all ethanol treated groups according to body weights

Effect of ethanol on body length

The results of body length according to the effect of different concentrations of ethanol are given in (Table $2 \&$ Figure 2). Its results revealed that different concentrations of ethanol showed significantly effect on group B and group C Chicks embryo but showed no significantly effect on the others groups embryos according to the comparison with the control group Chicks embryos.

Table 2. The Mean \pm SEM of body length of 7th-day embryo at different levels of alcohol concentration

\begin{tabular}{|c|c|c|}
\hline \multicolumn{3}{|c|}{ Body Length(cm) vs ethanol concentrations } \\
\hline Groups & Ethanol concentration & SEM \\
\hline Control & 00 & $3.45 \pm 0.09$ \\
\hline A & $5 \%$ & $2.95 \pm 0.28$ \\
\hline B & $10 \%$ & $2.18 \pm 0.27^{*}$ \\
\hline C & $15 \%$ & $2.2 \pm 0.16^{*}$ \\
\hline
\end{tabular}

$*=$ Significant, $\mathrm{p}<0.05$ 


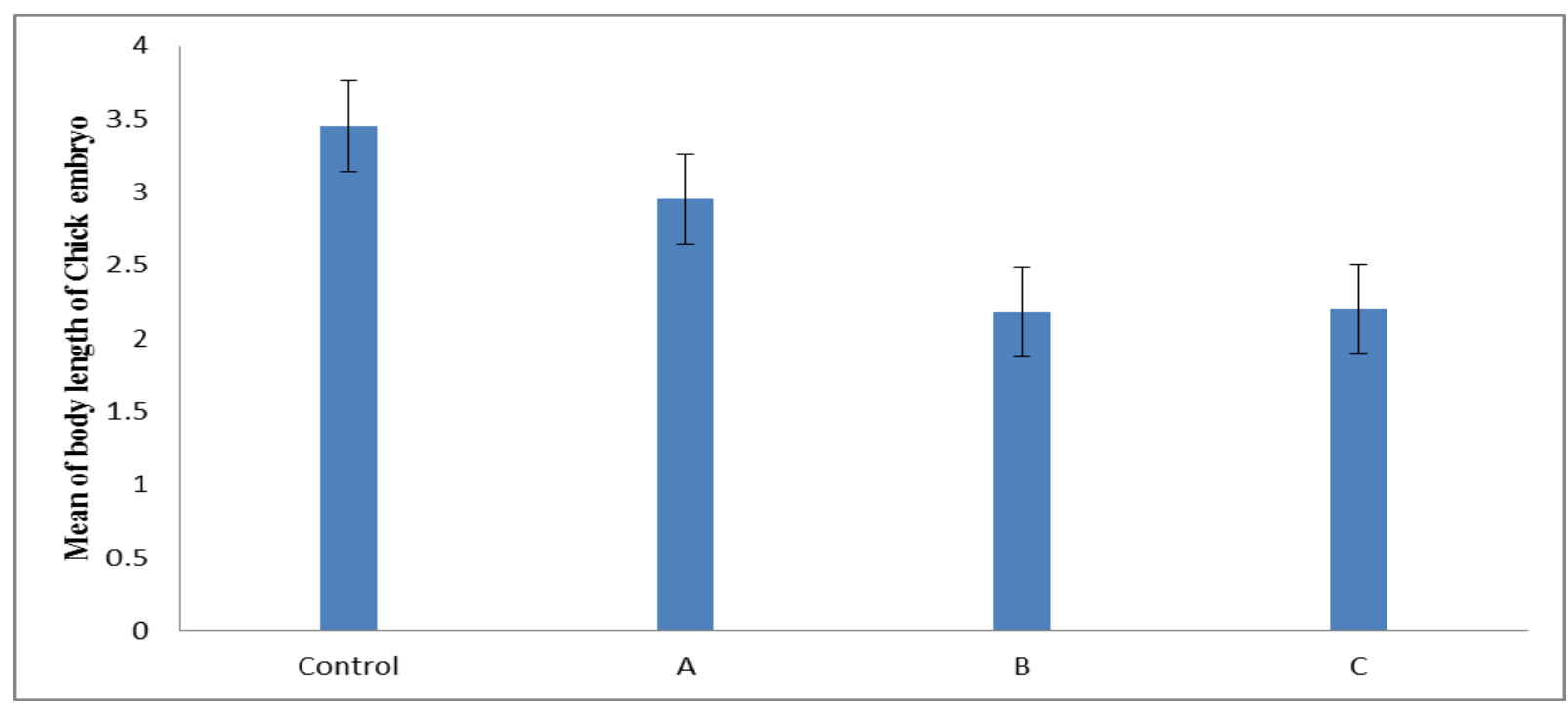

Figure 2. Comparison of Mean \pm SEM of all ethanol treated groups according to body length

\section{Effect of ethanol on wings length}

The means values of wings length of Chicks embryo according to the effect of different concentrations of ethanol are given in (Table $3 \&$ Figure 3$)$. The results of that mean values revealed that different concentrations of ethanol showed no significant effect on all the groups according to the comparison with the control group embryos.

Table 3. The Mean \pm SEM of wings length of 7th-day embryo at different levels of alcohol concentration

\begin{tabular}{|c|c|c|}
\hline \multicolumn{3}{|c|}{ Wings Length $(\mathbf{m m})$ vs. ethanol concentration } \\
\hline Groups & Ethanol Concentrations & SEM \\
\hline Control & 00 & $8.50 \pm 0.29$ \\
\hline A & $5 \%$ & $6.00 \pm 1.08$ \\
\hline B & $10 \%$ & $5.75 \pm 0.63$ \\
\hline C & $15 \%$ & $7.25 \pm 0.63$ \\
\hline
\end{tabular}

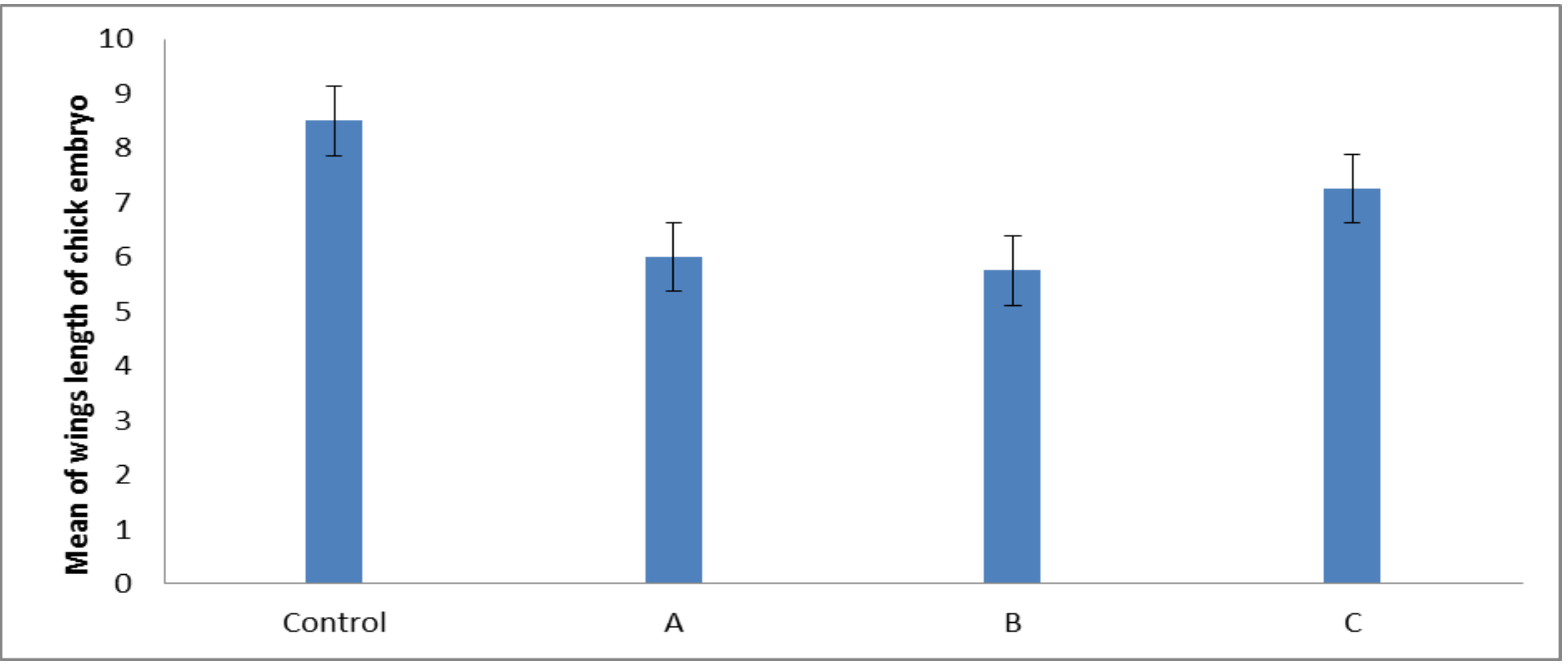

Figure 3. Comparison of Mean \pm SEM of all ethanol treated groups according to wings length 
Effect of ethanol on hind limbs length

The results of means values of hind limbs length according to the effect of different concentrations of ethanol are given in (Table 4 \& Figure 4). According to that results the concentrations of ethanol showed no significant effect on hind limbs length of Chick embryos of all groups concerning the comparison with control group Chicks embryos.

Table 4. The Mean \pm SEM of hind limbs length of the 7th-day embryo at different concentration

\begin{tabular}{|c|c|c|}
\hline \multicolumn{3}{|c|}{ Hind Limb Length (cm) vs. ethanol concentrations } \\
\hline Groups & Ethanol Concentration & SEM \\
\hline Control & 00 & $1.03 \pm 0.03$ \\
\hline A & $5 \%$ & $1.26 \pm 0.11$ \\
\hline B & $10 \%$ & $2.93 \pm 1.69$ \\
\hline C & $15 \%$ & $1.25 \pm 0.10$ \\
\hline
\end{tabular}

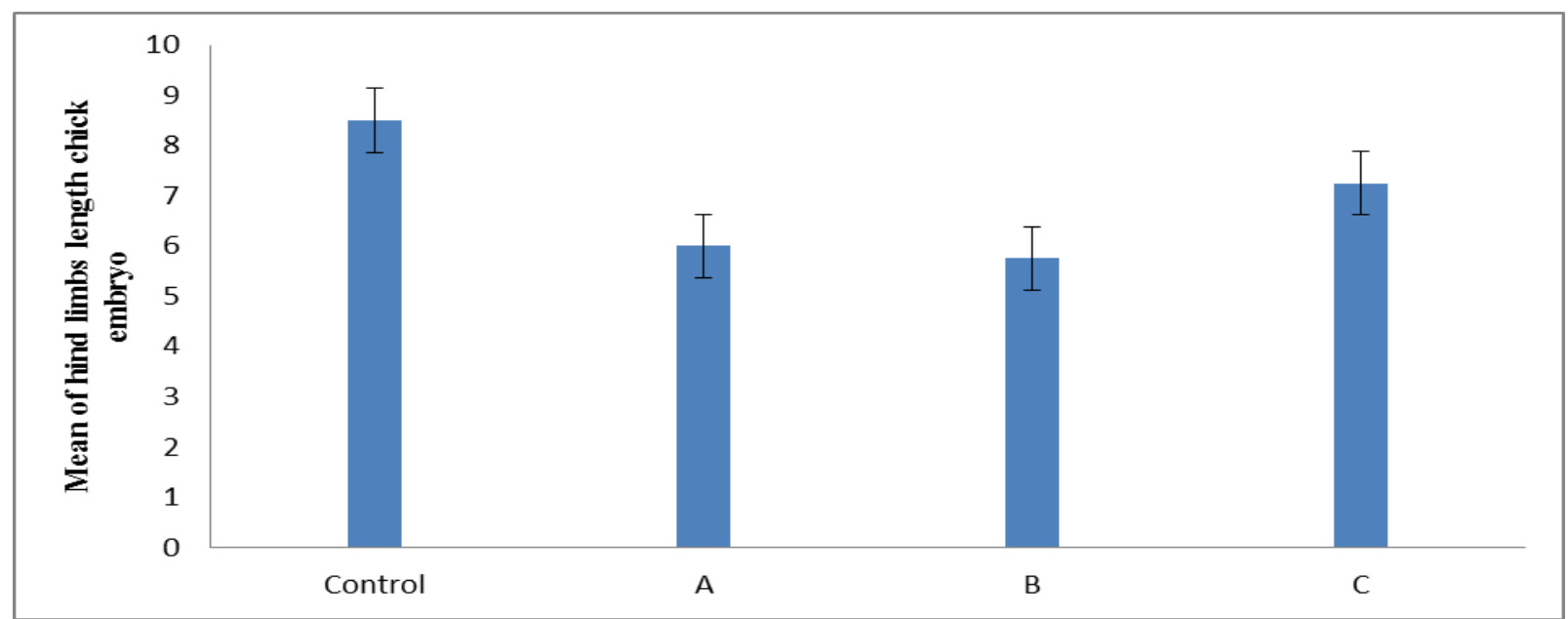

Figure 4. Comparison of Mean \pm SEM of all ethanol treated groups according to hind limb length

\section{The most frequent abnormalities}

The most frequent abnormalities among body weight, body length, wings length and hind limbs length of the entire group embryo were calculated concerning the control group. The body weight of the group with $5 \%$ ethanol exposure was found reduced while at $15 \%$ the hind limbs length of all the groups concerning control showed a high rate of abnormality. There was no significant effect on body length and wing length abnormalities regarding all groups (Table 5).

Table 5. Frequency Percentages of particular developmental defects in seven-day-old chick embryos

\begin{tabular}{|c|c|c|c|c|}
\hline \multicolumn{5}{|c|}{ Percentage reduction values among abnormalities concerning control } \\
\hline Groups & $\begin{array}{c}\text { Body Weight } \\
(\boldsymbol{\%})\end{array}$ & Body Length (\%) & Wing Length (\%) & Hind Limbs Length (\%) \\
\hline A & -3.61 & 14.49 & 29.41 & -22.93 \\
\hline B & 24.43 & 36.96 & 32.35 & -185.37 \\
\hline C & 21.31 & 36.23 & 14.71 & -21.95 \\
\hline
\end{tabular}




\section{Hatchability characteristics}

The newly hatched chicks of all groups exhibited diverse behavioral variations at different levels of ethanol exposure. In almost $90 \%$ of newly hatched chicks of the control group were active and healthy while $10 \%$ were taken out through aided hatching and displayed reduced mobility. A total of $33 \%$ chicks of group A and B showed poor balance, staggering wobbly gait. While $56 \%$ of chicks displayed reduced mobility and were inept at standing straight. On gross inspection of group $\mathrm{C}$, it was noticed that there was the failure of withdrawal of yolk sac into the abdominal cavity in the experimental chicks.

\section{Day of hatching}

Remaining fours (4) eggs from all of the groups were observed till hatching few of them was hatched on day 21 and few on day 22. All remaining four (4) eggs were hatched on 21 days, three eggs of group A, two eggs of group $\mathrm{B}$ and one egg of group $\mathrm{C}$ also hatched on day 21 . The remaining eggs like as 1 of group A, 2 of group B and 3 of group $\mathrm{C}$ were hatched on day 22 which are given in (Table 6) (Figure 5).

.Table 6. Day of hatching among ethanol-exposed groups

\begin{tabular}{|c|c|c|c|}
\hline \multicolumn{4}{|c|}{ Day of Hatching } \\
\hline Groups & Day 21 & Day 22 & Total \\
\hline Control & 4 & 0 & 4 \\
\hline A & 3 & 1 & 4 \\
\hline B & 2 & 2 & 4 \\
\hline C & 1 & 3 & 4 \\
\hline
\end{tabular}

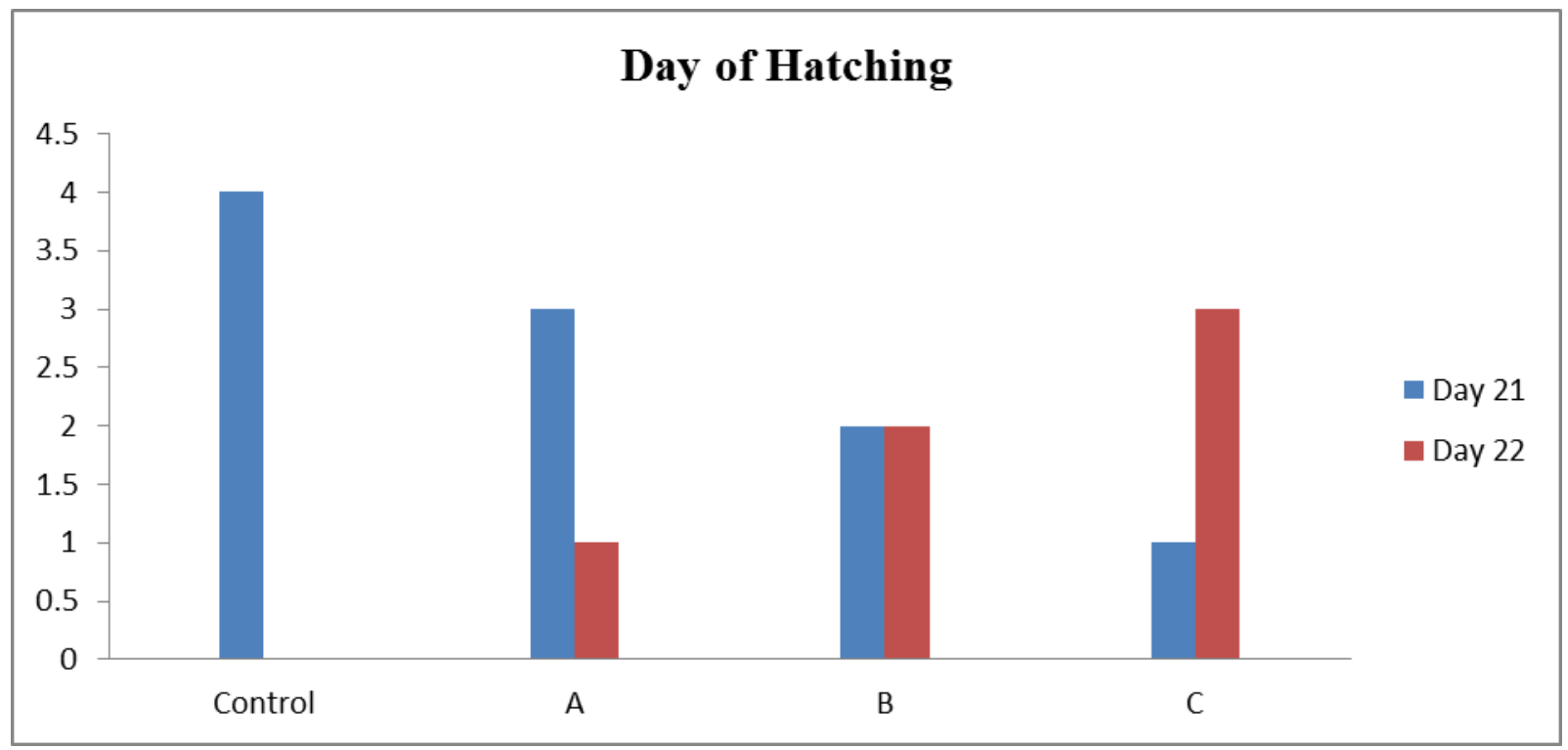

Figure 5. Day of Hatching according to different levels of alcohol exposure

\section{Mode of hatching}

There were few of the embryos produced normal at the time of hatchability, and few were abnormal. All embryos of the control group and group A were normal, two embryo of group B and one embryo of group C were also normal. But two embryo of group B and three embryo of group $\mathrm{C}$ were produced abnormal which are given in (Table $7 \&$ Figure 6). 
Table 7. Mode of hatching among ethanol-exposed groups

\begin{tabular}{|c|c|c|c|}
\hline \multicolumn{4}{|c|}{ Mode of Hatching } \\
\hline Groups & Normal & Assisted & Total \\
\hline Control & 4 & 0 & 4 \\
\hline A & 4 & 0 & 4 \\
\hline B & 2 & 2 & 4 \\
\hline C & 1 & 3 & 4 \\
\hline
\end{tabular}

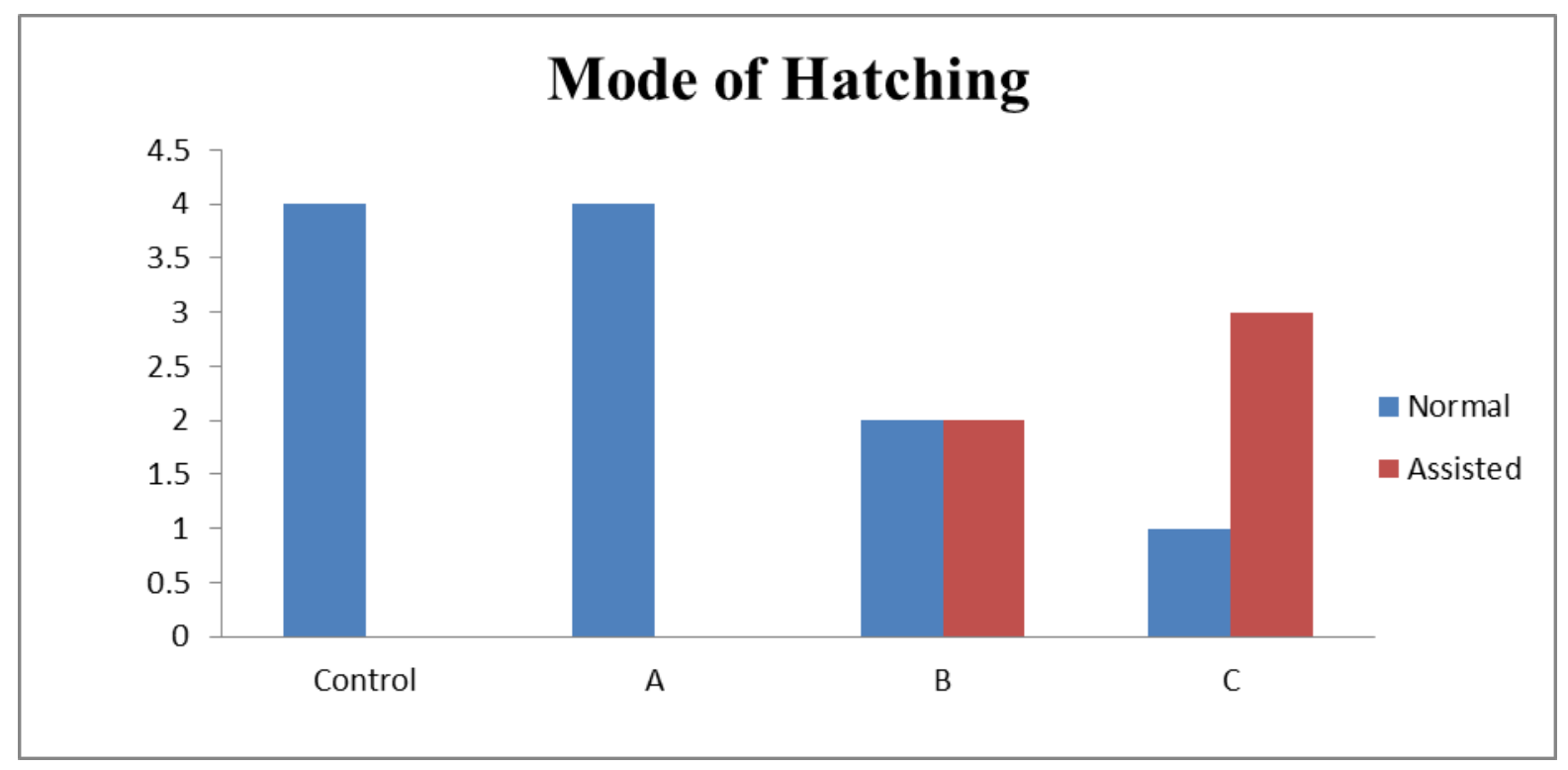

Figure 6. Mode of hatching according to different levels of alcohol exposure

\section{Discussion}

In this study ethanol effects of different concentrations, i.e. $00 \%, 5 \%, 19 \%, 15 \%$ on chicken embryo early developmental stages, their characteristics viz body weight, body length, wings length, and hind limbs length were observed. Eggs were divided into four groups, i.e. group Control, group A, group B and group $\mathrm{C}$ concerning ethanol concentration respectively. There was no significant $(\mathrm{P}>0.05)$ ethanol effect on weight, length of wings and hind limbs of the chick embryo of all groups vs. control group. Lower no of the abnormal embryo was found in all groups compared to the control group. Ethanol effects the chick embryo body length significantly $(\mathrm{P}<0.05)$ in group $\mathrm{B}$ and group $\mathrm{C}$ with concentration $19 \%$ and $15 \%$ respectively. It was in line with the findings [10-12]. They described the ethanol effect significantly on NCC of chick embryo in early stage and caused many abnormal changes compared to control. Our findings are similar to Fetal alcohol syndrome (FAS) on ocular anomalies reported $[2,13]$. They found ethanol had adverse effects on the retinal development and caused blindness during the early stage of the chick embryo. Giles et al. reported the ethanol impact on Central Nervous system during the early stage of chick embryo and caused FAS [14]. Findings of this study are highly correlated with the findings by Mason et al. (2012) regarding the effect of ethanol on facial morphology [15]. Memon and Kamran reported that ethanol effect significantly on the heart chambers and become a cause of FAS supports the finding of this study [16, 17]. FAS reported by Kiecker and Boronat are in line with this study [18]. They described ethanol cause a significant effect on craniofacial development in chick embryo 
during their early stage. Measurements were taken for embryo body length, wing bud length and leg bud length over a course of twenty one (21) days on a sample of 12-hours embryos that were exposed to four selected ethanol concentrations; concentration first is control in which no injection of ethanol, concentration second is $(5 \%)$, concentration third is $(10 \%)$ and concentration fourth is $(15 \%)$ [3].

The developmental and general growth effects as well as effects on skeletal muscles were studied by exposing the embryos chicks to single doses of $5 \%, 10 \%$, and $15 \%$ ethanol. There a significant hindrance was found in head circumference, crown-rump length, and body weight in ethanol-exposed chicks. These parameters were compared with results of control groups, and it was noticed that this growth retardation was linked with a substantial and proportionate decline in the weight of skeletal muscles [19]. In this research, $56 \%$ of chicks presented reduced mobility and were inept at standing upright. Additional possible cause of hypo-activity could be alcohol removal. The researchers exposed male Sprague Dawley rats to ethanol vapors for 12 or 14 days and then examined their locomotion activity. Hypo-activity appeared promptly in rats during ethanol removal. Extraction induced hypo-activity was noticed in previous studies directed on various species of rats after ethanol intake through various routes $[20,21]$. In the present study, the experimental group $\mathrm{C}$ exhibited a major failure of yolk sac withdrawal into the abdominal cavity. Generally, yolk sac starts withdrawing into the abdominal cavity at 19th day and this withdrawal is finished at day 20 [22]. There a substantial growth delay was found in these chicks, which is in agreement with our study. In a previous study, chick embryos were grown in shell-less cultures with supplementation of a single dose of $50 \%$ ethanol. Ethanol considerably enhanced the mortality rate and encouraged the growth impedance [23]. Experimental studies on various species revealed that oxidative stress, apoptosis, distorted cell cycle, blocked DNA and protein synthesis are some of the significant causes of ethanol persuaded malformations [24, 25]. No notable anomalies were found in live and dead chick embryos of group A and B in the experimental group, which demonstrated that both increase period of exposure and enhanced embryonic age increases the risk of developmental defects and growth hindrance in developing chick embryos.

\section{Conclusion}

In this study we found that the ethanol exposure enhanced the risks of developmental fetus defects with increased embryonic age, detained hatching and more assisted hatchings. Newly hatched chicks, which were exposed to alcohol exhibited reduced locomotion activity and poor balance which may be observed to damage skeletal muscle and central nervous system in growing embryos. These outcomes might have significance concerning the possible developmental effects of alcohol misuse at various stages of human pregnancy and the devising proper advice and involvement policies.

\section{Authors' contributions}

Conceived and designed the experiments: $\mathrm{G}$ Afzal, S Kiran \& G Mustafa, Performed the experiments: S Ali, H M Arshad \& J Majeed, Analyzed the data: H I Ahmad \& T Ruby, Contributed materials/ analysis/ tools: S Ali, S Hameed, M Nazir \& R Amjad, Wrote the paper: H I Ahmad \& G Afzal.

\section{Acknowledgment}

Authors are thankful to anonymous reviewers for critical review of the manuscript.

\section{References}

1. Ren J, Wold LE, Natavio M, Ren BH, Hannigan JH \& Brown RA (2002). Influence of prenatal alcohol exposure on myocardial contractile function in 
adult rat hearts: role of intracellular calcium and apoptosis. Alcohol and Alcoholism 37: 30-37.

2. Guerri C \& Pascual M (2017). Effects of Alcohol on Embryo/Fetal Development. In Reproductive and Developmental Toxicology (Second Edition), Elsevier. pp 431-445.

3. Zaza M (2015). Testing the Teratogenic Effects of Alcohol on Chicken Embryo Growth.

4. Burton DF, Zhang C, Boa-Amponsem O, Mackinnon S \& Cole GJ (2017). Long-term behavioral change as a result of acute ethanol exposure in zebrafish: Evidence for a role for sonic hedgehog but not retinoic acid signaling. Neurotoxicol and Teratol 61: 66-73.

5. Battistoni M, Mercurio S, Ficetola GF, Metruccio FC, Menegola E \& Pennati R (2018). The Ascidian Embryo Teratogenicity assay in Ciona intestinalis as a new teratological screening to test the mixture effect of the co-exposure to ethanol and fluconazole. Environ Toxicol and Pharmacol 57: 7685.

6. Giles S, Boehm P, Brogan C \& Bannigan J (2008). The effects of ethanol on CNS development in the chick embryo. Reprod Toxicol 25: 224-230.

7. Andréasson S, Chikritzhs T, Dangardt F Holder H, Naimi $\mathrm{T}$ \& Stockwell $\mathrm{T}$ (2014). Evidence about health effects of "moderate" alcohol consumption. Alcohol and Society 2014: 6.

8. Jung ME \& Mallet RT (2018). Intermittent hypoxia training: powerful, non-invasive cerebroprotection against ethanol withdrawal excitotoxicity. Respirat physiol neurobiol 256: 67-78.

9. Mok K M, Bahr $\mathrm{R} \&$ Krosshaug $\mathrm{T}$ (2017). The effect of overhead target on the lower limb biomechanics during a vertical drop jump test in elite female athletes. Scandinavian J Med Sci Sports 27: 161-166.

10. Flentke GR \& Smith SM (2017). The avian embryo as a model for fetal alcohol spectrum disorder. Biochem Cell Bio 96: 98-106.

11. Smith SM, Garic A, Flentke GR \& Berres ME (2014). Neural crest development in fetal alcohol syndrome. Birth Defects Research Part C: Embryo Today: Reviews 102: 210-220.

12. Fernandes RM, Nascimento PC, Bittencourt LO, de Oliveira ACA, Puty B, Leão LKR, Fernandes LMP, Cartágenes S, Maia CSF \& Lima RR (2018). Chronic ethanol forced administration from adolescence to adulthood reduces cell density in the rat spinal cord. Tissue and Cell 55: 77-82.

13. Holahan CJ, Brennan PL, Schutte KK, Holahan CK, Hixon, JG \& Moos RH (2018). Drinking Level Versus Drinking Pattern and Cigarette Smoking Among Older Adults. Alcoholism: Clin. Experiment Res 42: 795-802.

14. Gilles M, Otto H, Wolf IA, Scharnholz B, Peus V, Schredl M, Sütterlin MW, Witt SH, Rietschel M \& Laucht M (2018). Maternal hypothalamuspituitary-adrenal (HPA) system activity and stress during pregnancy: Effects on gestational age and infant's anthropometric measures at birth. Psychoneuroendocrinol 94: 152-161.

15. Uhari-Väänänen $J$, Raasmaja $A$, Bäckström P, Oinio V, Carroll FI, Airavaara M, Kiianmaa K \& Piepponen $P$ (2018). The $\kappa$-opioid receptor antagonist JDTic decreases ethanol intake in alcohol-preferring AA rats. Psychopharmacol 235: 1581-1591.

16. Memon SF, Lewis E, Pembroke JT \& Chowdhry BS (2017). A novel interfibre light coupling sensor probe using plastic optical fibre for ethanol concentration monitoring at initial 
production rate. In Optical Fiber Sensors Conference (OFS) 2017 25th pp. 1-4, IEEE.

17. Al-Wajeeh NS, Hajerezaie M, Noor SM, Halabi MF, Al-Henhena N, Azizan AHS, Kamran S, Hassandarvish P, Shwter AN \& Ali HM (2016). The gastro protective effects of Cibotium barometz hair on ethanol-induced gastric ulcer in Sprague-Dawley rats. BMC vet res 13 : 27.

18. Kiecker C (2016). The chick embryo as a model for the effects of prenatal exposure to alcohol on craniofacial development. Development Biol 415: 314-325.

19. Chaudhuri JD (2004). Effect of a single dose of ethanol on developing skeletal muscle of chick embryos. Alcohol 34: 279-283.

20. Doremus-Fitzwater TL \& Spear LP (2007). Developmental differences in acute ethanol withdrawal in adolescent and adult rats. Alcoholism: Clin. Experiment Res 31: 1516-1527.
21. An L, Zhao T \& Li Y (2015). Carbonneutral sustainable energy technology: direct ethanol fuel cells. Ren Sustain Energy Rev 50: 1462-1468.

22. Haque A \& Khan MY (2016). Does folinic acid ameliorates retinoic acid induced teratogenicity in chick embryo? JPMA. The Journal of the Pakistan Medical Association 66: 302-305.

23. Shabtai Y \& Fainsod A (2017). Competition between ethanol clearance and retinoic acid biosynthesis in the induction of fetal alcohol syndrome. Biochem. Cell Biol 96: 148-160.

24. Schambra UB, Nunley K, Harrison TA \& Lewis CN (2016). Consequences of low or moderate prenatal ethanol exposures during gastrulation or neurulation for open field activity and emotionality in mice. Neurotoxicol. teratol 57: 39-53.

25. Wetherill L, Foroud $\mathrm{T} \&$ \& Goodlett $\mathrm{C}$ (2018). Meta-Analyses of Externalizing Disorders: Genetics or Prenatal Alcohol Exposure? Alcoholism: Clin and Experi Res 42: 162-172. 